\title{
Metastatic Squamous cell carcinoma of small bowel with occult primary presented as small bowel obstruction
}

\author{
Sridhar Reddy M.*, Naresh M., Alok Rath
}

Department of General Surgery, CARE Hospitals, Banjara Hills, Hyderabad, Telangana, India

Received: 10 February 2018

Accepted: 13 March 2018

\section{*Correspondence:}

Dr. Sridhar Reddy M.,

E-mail: sridhar.muthadi@gmail.com

Copyright: ( ) the author(s), publisher and licensee Medip Academy. This is an open-access article distributed under the terms of the Creative Commons Attribution Non-Commercial License, which permits unrestricted non-commercial use, distribution, and reproduction in any medium, provided the original work is properly cited.

\begin{abstract}
Metastatic lesions in the small bowel are more common than primary lesions and account for $0.5 \%$ of all small intestinal malignancies. Most common is malignant melanoma followed by adenocarcinoma from pancreas, colon or stomach; and squamous cell carcinoma from lung, cervix, esophagus, skin, penis, ovary, pancreas and gallbladder. Gastrointestinal involvement as a manifestation of metastatic squamous cell carcinoma with unknown primary is relatively uncommon with very few being reported in the literature. The most common presenting symptom of small bowel lesions is subacute or acute obstruction and less commonly, bowel perforation, abdominal pain or hemorrhage. It is a diagnosis which dawns upon after the surgery when histopathology proves the same and therefore a thorough workup to establish the primary starts after the bailout procedure. Its clinical course is aggressive, characterized by a short preclinical history, resistance to chemotherapy, and overall dismal prognosis with a median life expectancy of 6 to 9 months as it represents a disseminated disease.
\end{abstract}

Keywords: Metastatic SCC, Small bowel obstruction

\section{INTRODUCTION}

Malignant tumors of the small intestine (SI) are very rare compared to other gastro-intestinal organs; fewer than $2 \%$ of all GI malignancies originate in the small bowel. ${ }^{1,2}$ Duodenum is the commonest site followed by jejunum and ileum. However, metastatic cancers of the small bowel are more frequent than primary SI cancers., ${ }^{3,4}$ Primary carcinomas of small intestine comprise $0.1-1.3 \%$ of all GI malignancies of which approximately $30 \%$ to $50 \%$ are adenocarcinomas, $25 \%$ to $30 \%$ are carcinoids, and $15 \%$ to $20 \%$ are lymphomas. ${ }^{2}$ Squamous cell carcinomas of the SI, are exceptionally rare and are more likely to be metastatic. Most frequently metastasizing tumours are melanoma, next is adenocarcinoma followed by squamous cell carcinoma. Most common site for primary squamous cell carcinoma is lungs followed by cervix and uterus; oesophagus, head and neck. Most common site for primary adenocarcinoma is kidney followed by lung, breast, colon, adrenals and stomach. Primary site for melanoma is skin. It is difficult to differentiate primary from metastatic lesion of small bowel owing to the rarity of occurrence. Usually they present with GI bleeding, obstruction, perforation or abdominal lump. We report a case of metastatic squamous cell carcinoma of small bowel manifesting as acute intestinal obstruction.

\section{CASE REPORT}

A 59-year-old hypertensive female was evaluated in the outpatient with history of intermittent central abdominal pain for 20 days. Onset of pain was few hours after intake of food and was associated with bilious vomiting. Pain decreased in intensity after vomiting. She gave history of loss of appetite and increasing constipation. She 
underwent abdominal hysterectomy a year ago for abnormal uterine bleeding. An USG of abdomen and pelvis revealed post hysterectomy status with minimal ascites and mildly dilated small bowel loops with sluggish to and fro peristalsis. An erect and supine X-ray of abdomen (Anteroposterior view showing both domes of diaphragm) revealed dilated small bowel loops and gas filled portions of colon with no significant air fluid levels.

She was diagnosed as subacute intestinal obstruction; managed symptomatically with antispasmodics, liquid diet, laxatives, enemas and anti-emetics. She showed slight improvement for a couple of weeks when she was admitted in emergency room with complaints of continuous pain, abdominal distension, vomiting and obstipation. On examination, she was conscious, well oriented and hemodynamically stable. Abdomen was soft, distended with mild tenderness all over abdomen and sluggish bowel sounds. There was a pfannensteil scar with no evidence of incisional hernia. On evaluation, erect X-ray abdomen showed multiple air fluid levels with dilated small bowel loops.
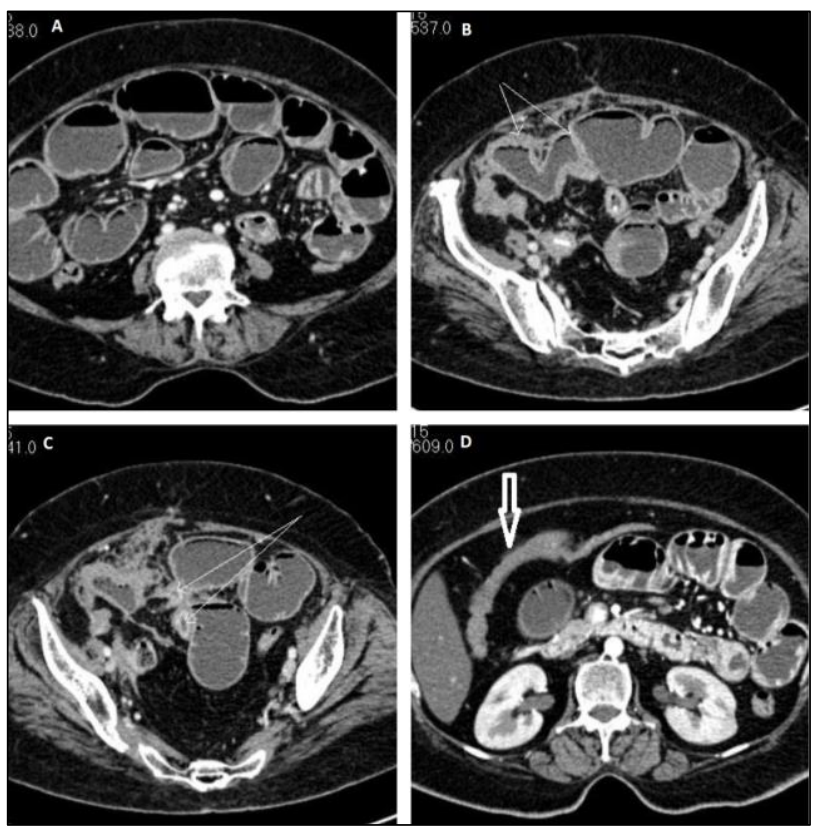

Figure 1: CT scan images; (a) Dilated small bowels with air-fluid levels; (b) Thick walled adherent small bowel; (c) Collapsed adherent small bowel loops with fat stranding; (d) Collapsed large bowel.

A CECT abdomen showed dilated small bowel loops with transition point at distal ileum, adjacent bowel wall thickening and irregular enhancement on contrast films suggestive of small bowel obstruction (Figure 1). Patient was optimized and taken up for exploratory laparotomy. On exploration, dense adhesions between loops of terminal ileum, mesentery, caecum, omentum, parieties and urinary bladder was noticed (Figure 2). Colon was collapsed; proximal ileal and jejunal loops were dilated. Adhesiolysis attempted but abandoned as the adhesions were dense. A proximal diverting loop ileostomy was performed, and tissue sent for biopsy.
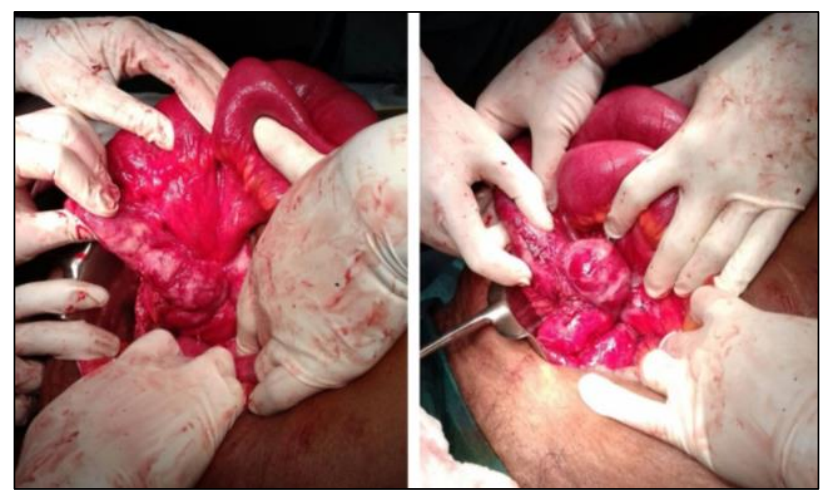

Figure 2: Intra operative image showing densely adherent small bowel loops.

On the second postoperative day, she was extubated, weaned off from ionotropes but patient continued to have tachycardia and fever. Her condition deteriorated further, and her laboratory parameters showed pancytopenia and features of sepsis.

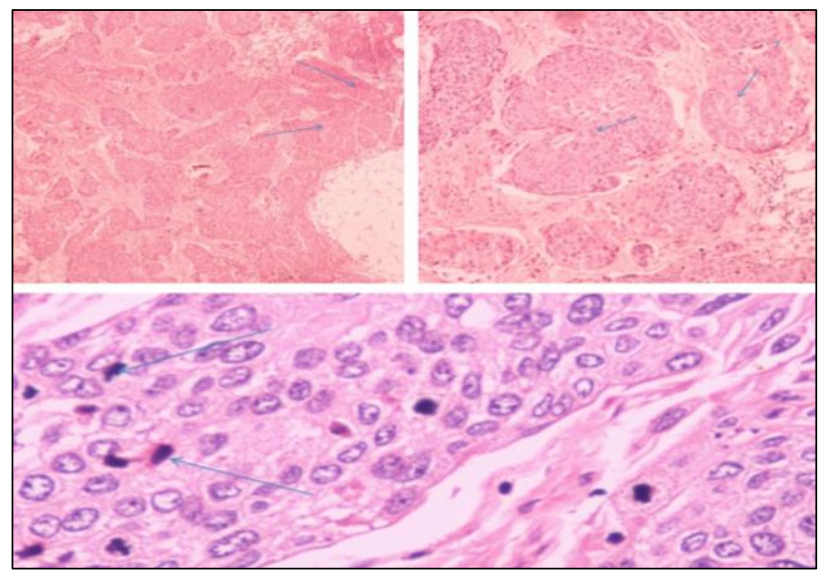

Figure 3: HPE showing (A) Infiltration by squamous cell islands; (B) Tumor cells as nests in adipose tissue; (C) Mitosis.

However, despite all efforts, she succumbed on the fourth postoperative day. Histopathology report showed metastatic deposits of non-small cell carcinoma favoring morphological features of squamous cell carcinoma (Figure 3).

\section{DISCUSSION}

Gastrointestinal metastases are an extremely rare occurrence in the scenario of distant metastases. Small bowel is the most common site of gastrointestinal metastases. Clinically occult primary malignancy with small bowel metastases is an enigma and a review of literature establishes its rarity. Metastatic lesions in the small bowel are more common than primary lesions and 
account for $0.5 \%$ of all small intestinal malignancies. ${ }^{5}$ Malignant melanoma is the most common extragastrointestinal primary to metastasize to the small bowel, followed by adenocarcinoma from pancreas, colon or stomach, but metastatic squamous cell carcinoma (SCC) is extremely rare, most common primary site being lung, other sites being esophagus, skin, cervix, penis, ovary, pancreas and gallbladder. ${ }^{6-9}$

The diagnostic criteria for secondary small bowel SCC include primary SCC or history of excision of SCC from the lung or other organs. Histopathologically, secondary SCC generally presents as cancer invasion in the submucosal, muscular layer and serosa. ${ }^{10}$ Secondary SCC does not usually involve the glandular epithelium. ${ }^{10}$

The most common presenting symptom of small bowel lesions is a partial or complete bowel obstruction and less commonly, bowel perforation, abdominal pain or hemorrhage. ${ }^{11,12}$ Time variation between the primary and the manifestation of metastatic lesions is reported in the literature ranging from being synchronous to metachronous with time interval ranging from 2 to 13 years. ${ }^{11-13}$ Clinical presentations reported are varied and the overall long-term prognosis of cases with small bowel metastases is extremely poor.

Discovery of metastatic carcinoma of an unknown primary origin necessitates a careful clinical workup and complete histopathological examination, as well as concomitant imaging studies. However, preemptive diagnosis of small bowel involvement before perforation or obstruction is difficult, as the vast majority of patients initially presents with an acute abdomen or peritonitis and often require emergency abdominal surgery. Radiologic imaging studies can be useful in detecting the primary site. Studies have shown that either a PET/CT or a CT alone is sufficient in detecting a primary tumor in the majority. Immunohistochemical (IHC) staining of metastatic tissue can be useful in suggesting the primary site.

\section{CONCLUSION}

In our experience, the patient presented with a small bowel obstruction with history of hysterectomy in the remote past. Corroborative documents regarding details of surgery and histopathology were not available. The patient deteriorated fast in the postoperative period and expired on the fifth postoperative day in agreement with the evidence available in literature which underscores the dismal prognosis.

Funding: No funding sources Conflict of interest: None declared

Ethical approval: Not required

\section{REFERENCES}

1. Barclay TH, Schapira DV. Malignant tumors of the small intestine. Cancer. 1983;5:878-81.

2. Gill SS, Heuman DM, Mihas AA. Small intestinal neoplasms. J Clin Gastroenterol. 2001;33:267-82.

3. Disibio G, French SW. Metastatic patterns of cancers: results from a large autopsy study. Arch Pathol Lab Med. 2008;132:931-9.

4. Thomas RM, Sobin LH. Gastrointestinal cancer. Cancer. 1995;75(1):154-70.

5. Dwivedi RC, Kazi R, Agrawal N, Chisholm E, St Rose S, Elmiyeh B, et al. Comprehensive review of small bowel metastasis from head and neck squamous cell carcinoma. Oral Oncol. 2010;46:3305 .

6. Kanthan R, Gomez D, Senger JL, Kanthan SC. Endoscopic biopsies of duodenal polyp/mass lesions: A surgical pathology review. J Clin Pathol. 2010;63:921-5.

7. Wang M, Patel J, Casey TT, Kieffer R, Dunn GD. Metastatic squamous cell carcinoma from the esophagus occurring as small bowel obstruction. South Med J. 1985;78:884-6.

8. Yamada H, Akahane T, Horiuchi A, Shimada R, Shibuya H, Hayama $T$, et al. A case of lung squamous cell carcinoma with metastases to the duodenum and small intestine. Int Surg. 2011;96:176-81.

9. Terada T. Malignant tumors of the small intestine: a histopathologic study of 41 cases among 1312 consecutive specimens of small intestine. Int J Clin Exp Pathol. 2012;5:203-9.

10. Yamada T, Yagi S, Tatsuzawa Y, Fujioka S, Sato H, Kitagawa $S$, et al. Small intestinal metastasis from esophageal carcinoma associated with small intestinal obstruction: report of a case. Surg Today. 1996;26:800-2.

11. Gurian L, Ireland K, Petty W, Katon R. Carcinoma of the cervix involving the duodenum: Case report and review of the literature. J Clin Gastroenterol. 1981;3:291-4.

12. Mathur SK, Pandya GP. Solitary metastatic malignant stricture of the ileum: A rare cause of small bowel obstruction (a case report). J Postgrad Med. 1984;30:186-8.

13. Misonou J, Natori T, Aizawa M, Jou B, Tamaki A, Ogasawara M. Stage (Ia) cervical cancer recurring 13 years after hysterectomy and causing small intestinal perforation. A case report with a review of the literature. Acta Pathol Jpn. 1988;38:225-34.

Cite this article as: Reddy SM, Naresh M, Rath A. Metastatic Squamous cell carcinoma of small bowel with occult primary presented as small bowel obstruction. Int Surg J 2018;5:1584-6. 